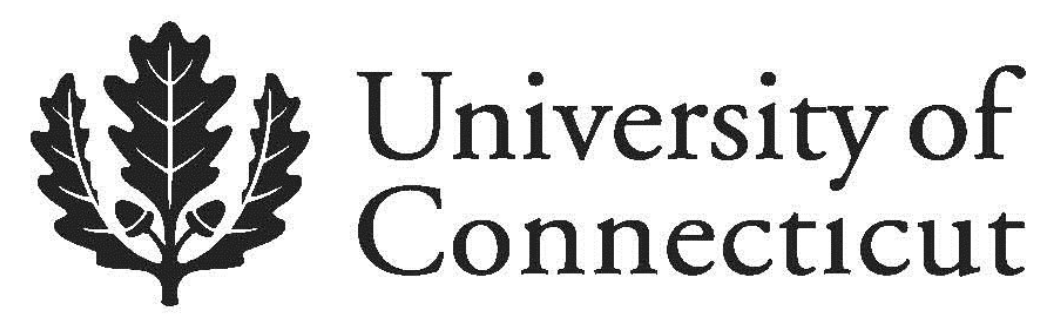

Department of Economics Working Paper Series

Focusing Law Enforcement When Offenders Can Choose Location

Tim Friehe

University of Bonn

Thomas J. Miceli

University of Connecticut

Working Paper 2014-15

July 2014

365 Fairfield Way, Unit 1063

Storrs, CT 06269-1063

Phone: (860) 486-3022

Fax: (860) 486-4463

http://www.econ.uconn.edu/

This working paper is indexed on RePEc, http://repec.org 


\title{
Focusing Law Enforcement When Offenders Can Choose Location
}

\author{
Tim Friehe* Thomas J. Miceli ${ }^{\dagger}$
}

July 15,2014

\begin{abstract}
This paper explores the advantages of focusing law enforcement on some locations when offenders can choose locations. The substitutability of different crimes from the offender's perspective is established as the key variable determining whether asymmetric enforcement is socially desirable. When it is easy for offenders to substitute crimes, focused law enforcement can be preferable only when the act imposes more harm in one location than in another (e.g., speeding in a residential or industrial area).
\end{abstract}

Keywords: crime, deterrence, focused enforcement, allocation of police

$J E L: K 42$

${ }^{*}$ University of Bonn, Center for Advanced Studies in Law and Economics, Adenauerallee 24-42, 53113 Bonn, Germany. CESifo, Munich, Germany. E-mail: tim.friehe@uni-bonn.de.

${ }^{\dagger}$ University of Connecticut, Department of Economics, 309 Oak Hall, 365 Fairfield Way, Storrs, CT 062691063. E-mail: Thomas.Miceli@UConn.edu.

We gratefully acknowledge the comments by Henrik Lando. Tim Friehe is thankful for the hospitality of the University of Connecticut, where this project was completed during a research visit. 


\section{Introduction}

Policing in practice is often asymmetric across space and target groups. For example, law enforcement authorities sometimes subscribe to so-called "hot spot" policing strategies that concentrate enforcement resources in few well-defined areas (Kleiman 2010). In such cases, it is usually assumed that the areas differ in their likelihood of crime occurring. Targeting of areas that are similar in their characteristics also occurs (e.g., when the police determine which highway to patrol). ${ }^{1}$ For optimal law enforcement, it is key to understand the scenarios in which focused law enforcement is socially optimal. In a related vein, private agents sometimes inform other individuals that enforcement is focused on some location. For example, some radio stations provide information about the location of speed cameras. An important question is to know in what contexts it is detrimental for society that potential offenders are informed about any asymmetry in law enforcement at different locations.

In a recent paper, Lando and Shavell (2004) (LS hereafter) examined how society should optimally allocate a fixed amount of enforcement resources across the set of potential offenders. Specifically, they asked whether it is ever desirable to focus all resources on a particular subgroup of offenders, for example, those residing in a particular region or possessing some discernible characteristic (e.g., drivers of red cars), rather than to spread the resources out uniformly. Their conclusion was that if resources are sufficiently constrained (i.e., below a critical threshold), then focusing all enforcement efforts on one subgroup is optimal. The intuition is that focusing enforcement allows achievement of the highest gain per unit of enforcement (e.g., per police officer) over some subset of offenders (the largest possible subset), thereby yielding a greater overall gain than settling for a lesser return per officer over the entire set.

This paper builds on the results established by LS by elaborating on the circumstances under which focused law enforcement may be optimal. A crucial assumption by LS is that offenders cannot respond to focused enforcement by changing their group membership. In some cases, this assumption makes perfect sense, for example, if membership in the targeted

\footnotetext{
${ }^{1}$ Police sting operations are a related strategy. See, for example, Hay (2005) and Miceli (2007).
} 
group depends on some inherent characteristic of potential offenders (e.g., male versus female). In other cases, however, individuals may have the ability to react to the announced policy; for example, if it involves only patrolling a certain highway, people could alter their routes. $^{2}$ We analyze a setting in which potential offenders can respond to focused law enforcement by undertaking their offense somewhere else (albeit at a cost), and show that this possibility mitigates the advantages of focusing enforcement at one location. ${ }^{3}$

The remainder of our paper is organized as follows. Section 2 describes the model used for our analysis. Section 3 presents our analysis and relates it to the one by LS. The final section concludes.

\section{The model}

Suppose that on a line of unit length, location A is set at 0 while location B is set at 1. Individuals are located uniformly on the line between 0 and 1 . Individuals choose, first, between a legal activity and an illegal activity, and, second, between conducting their activity at location $\mathrm{A}$ or location B. ${ }^{4}$ For example, the choice may be between, first, driving while obeying the speed limit and speeding, and, second, between itinerary A and B. The legal activity generates utility $v$. The gross benefit from undertaking the illegal activity is $b$, where $b \in[0, G]$ according to $F(b)$ with $G>v$ such that gross benefits from crime make offending profitable for some individuals when there is no effective law enforcement. Maintaining the assumption by LS, the distribution of benefits $F(b)$ applies irrespective of the individuals' location. There is a transportation cost $t x$ for somebody located at $x$ on the interval choosing to act at location $\mathrm{A}$, where $t / 2<v$ such that somebody located at $x=1 / 2$ finds tolerating the travel costs worthwhile given the benefit $v$. In the traffic example, for instance, location of the individual's residence and place of work may make itinerary A more convenient, when

\footnotetext{
${ }^{2} \mathrm{LS}$ recognize the relevance of this possibility in their discussion.

${ }^{3}$ To analyze this question, we make use of a transportation cost model that is similar to models that have been used to analyze competition in law enforcement between jurisdictions (see, e.g., Marceau 1997, Marceau and Mongrain 2011).

${ }^{4}$ There is no loss in generality in assuming only two locations since LS show that it is never desirable to divide the population of offenders into more than two subgroups.
} 
all else is held equal. ${ }^{5}$ The same logic applies to location $\mathrm{B}$, for which the transportation cost is $t(1-x)$.

Any offense at location $\mathrm{A}$ and $\mathrm{B}$ creates social harm, $h_{A} \geq h_{B}>0$. In order to deter offending, law enforcement authorities produce detection probabilities $p_{A}$ and $p_{B}$ at locations $\mathrm{A}$ and $\mathrm{B}$, respectively, and impose the fine $s$ upon detection. Like LS, we focus on the potential asymmetry in law enforcement and therefore consider $s$ to be exogenous. Law enforcement authorities at locations $\mathrm{A}$ and $\mathrm{B}$ coordinate their efforts, choosing the (potentially asymmetric) detection probabilities $p_{A}$ and $p_{B}$ that fulfill the budget constraint $P=p_{A}+p_{B} \cdot{ }^{6}$ That is, we assume that the level of resources used is proportional to the detection probability induced. For example, the number of people that can be checked by two policemen is twice the number of individuals that can be checked by one policeman. A uniform enforcement scheme would imply that the detection probability is $P / 2$ in location $\mathrm{A}$ and location $\mathrm{B}$. We also assume, without loss of generality, that $p_{A} \geq P / 2$, or that in a non-uniform enforcement scheme, resources are focused at location A.

\section{The analysis}

In this section, we will first describe how individuals make their decisions. Next, we analyze how law enforcement resources are optimally allocated between locations A and B. Finally, we relate our analysis more closely to that of LS.

\subsection{Individual decision-making}

Individuals determine the kind of activity, legal or illegal, and where to conduct the activity, location $\mathrm{A}$ or $\mathrm{B}$. We can distinguish individuals according to their level of realized gross criminal benefit $b \in[0, G]$ and their location $x \in[0,1]$ on the unit interval. At each location, we have to distinguish individuals who have drawn a small, intermediate or high level of gross benefit from the illegal activity from crime; that is, whether $b$ is in $\mathcal{C}_{0}=\left[0, b_{1}\right), \mathcal{C}_{1}=\left[b_{1}, b_{2}\right)$

\footnotetext{
${ }^{5}$ We assume that transportation costs are independent of the activity chosen.

${ }^{6}$ For simplicity, we assume that the constraint will be binding for the optimal allocation of funds, which will be true for sufficiently high levels of harm.
} 
or $\mathcal{C}_{2}=\left[b_{2}, G\right]$, where

$$
\begin{aligned}
& b_{1}=p_{B} s+v=\left(P-p_{A}\right) s+v \\
& b_{2}=p_{A} s+v
\end{aligned}
$$

using $p_{B}=P-p_{A}$ by fulfillment of the budget constraint. When law enforcement authorities choose uniform detection probabilities in location $\mathrm{A}$ and $\mathrm{B}$, then $b_{1}=b_{2}$. Otherwise, $b_{1}<b_{2}$ since resources are focused at location $\mathrm{A}$, and

$$
\frac{d b_{1}}{d p_{A}}=-s<0<\frac{d b_{2}}{d p_{A}}=s
$$

From (3), it is clear that there is a one-to-one substitution rate for the levels of deterrence $b_{1}$ and $b_{2}$.

Individuals in the set $\mathcal{C}_{0}$ are law-obedient in both locations because their gross benefits drawn according to the distribution $F(b)$ fall short of the sum of the benefits from the legal act $v$ and the minimum of the two expected sanctions. The split of this set on the unit interval occurs at $x_{0}$, such that individuals located at $x \leq x_{0}\left(x>x_{0}\right)$ undertake the legal activity at location $\mathrm{A}(\mathrm{B})$, where

$$
\begin{aligned}
& v-t x_{0}=v-t\left(1-x_{0}\right) \Leftrightarrow \\
& x_{0}=\frac{1}{2} .
\end{aligned}
$$

Individuals with $b \in \mathcal{C}_{1}$ (if that set exists) prefer crime over the legal activity only in location B, whereas the higher expected sanction at location A reverses the preference for location A. The split of this set occurs at $x_{1} \leq 1 / 2$, such that individuals located at $x \leq x_{1}$ select the legal activity at location $\mathrm{A}$ and individuals at $x>x_{1}$ undertake the illegal activity at location B, where

$$
\begin{aligned}
v-t x_{1} & =b-\left(P-p_{A}\right) s-t\left(1-x_{1}\right) \Leftrightarrow \\
x_{1}(b) & =\frac{1}{2}-\frac{b-\left(\left(P-p_{A}\right) s+v\right)}{2 t} \\
& =\frac{1}{2}-\frac{b-b_{1}}{2 t} .
\end{aligned}
$$


The level $x_{1}(b)$ (i.e, the location on the unit interval with the critical individual who is indifferent between the legal activity at location $\mathrm{A}$ and crime at location $\mathrm{B}$ ) is a function of the gross benefit from the illegal activity because individuals with $b \in \mathcal{C}_{1}$ compare the legal activity at location $\mathrm{A}$ to the offense at location $\mathrm{B}$. The form of $x_{1}$ leads to

$$
\frac{d x_{1}}{d p_{A}}=-\frac{s}{2 t}<0
$$

which is independent of $b$. In words, an increase in the level of the detection probability at location A increases the number of individuals with a given level of the gross benefit $b$ who are ready to offend in location $\mathrm{B}$, thereby decreasing the number of individuals choosing the legal act in location A. This marginal effect is critically influenced by the level of the transportation cost parameter $t$ because individuals find offending in the distant location $\mathrm{B}$ more appealing when traveling there is not very costly.

Finally, individuals with $b \in \mathcal{C}_{2}$ prefer offending to the legal activity in both locations, and for that reason constitute the set of hardcore criminals. The split of this set occurs at $x_{2} \leq 1 / 2$, such that individuals located at $x \leq x_{2}\left(x>x_{2}\right)$ offend in location $\mathrm{A}(\mathrm{B})$, where

$$
\begin{aligned}
b-p_{A} s-t x_{2} & =b-\left(P-p_{A}\right) s-t\left(1-x_{2}\right) \Leftrightarrow \\
x_{2} & =\frac{1}{2}-\frac{\left(2 p_{A}-P\right) s}{2 t} . \\
& =\frac{1}{2}-\frac{b_{2}-b_{1}}{2 t}
\end{aligned}
$$

This implies that $x_{2}$, in contrast to $x_{1}$, is not a function of the level of the gross benefit from crime. Moreover, we find that

$$
\frac{d x_{2}}{d p_{A}}=-\frac{s}{t}<0
$$

and $d x_{2} / d p_{A}=2 d x_{1} / d p_{A}<0$. The change in the critical level of $x$ dividing hardcore criminals at location $\mathrm{A}$ and at location $\mathrm{B}$ is greater the smaller is the level of the transportation cost parameter.

The split of individuals is illustrated in Figure 1 for the case in which $p_{A}>p_{B}$. Note that the level of $x_{1}$ is decreasing with the level of the gross benefits from crime (see (5)). We have that $x_{1}=x_{2}$ at $b=b_{2}, x_{1}>x_{2}$ when $b_{1}<b<b_{2}$, and $x_{1}=x_{0}$ at $b=b_{1}$. Individuals 


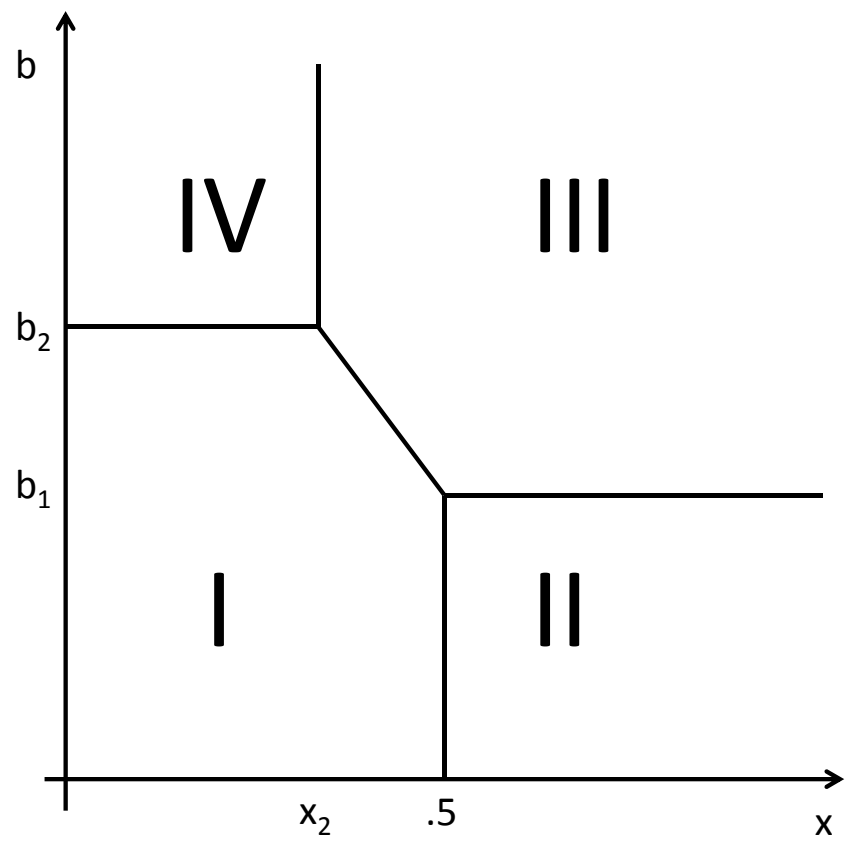

Figure 1: Split of population according to activity and location when $p_{A}>p_{B}$

in Region I (II) choose the legal activity at location A (B); individuals in Region III offend at location B; and individuals in Region IV offend at location A.

\subsection{The potential use of focused law enforcement}

The law enforcement authorities may focus law enforcement efforts on location A by setting

$p_{A} \in[P / 2, P]$ (i.e., the default option is uniform enforcement). We follow the literature (e.g., Polinsky and Shavell 2007) in assuming that policy makers maximize welfare defined as the summation of benefits less harm. Enforcement expenditures are given by the exogenous level $P$ and may be neglected in the following. In our context, we additionally incorporate the 
transportation costs, denoted $T C$, and arrive at welfare given by

$$
\begin{aligned}
W\left(p_{A}\right)= & v F\left(b_{1}\right)+\int_{b_{1}}^{b_{2}}\left[x_{1}(b) v+\left(1-x_{1}(b)\right)\left(b-h_{B}\right)\right] d F(b) \\
& \left.+\int_{b_{2}}^{G}\left[b-x_{2} h_{A}-\left(1-x_{2}\right) h_{B}\right)\right] d F(b)-T C
\end{aligned}
$$

Individuals with $b \in\left[0, b_{1}\right)$ choose the legal act. Individuals with $b \in\left[b_{1}, b_{2}\right)$ do not offend only when they choose location A (i.e., when they are located on the unit interval such that $\left.x \leq x_{1}(b)\right)$. Finally, individuals with $b \in\left[b_{2}, G\right]$ constitute the set of hardcore criminals, where the share $x_{2}$ offends in location $\mathrm{A}$. The transportation costs incurred by the individuals are given by

$$
T C\left(p_{A}\right)=\left(1-F\left(b_{2}\right)\right) t\left[\frac{1}{2}-x_{2}\left(1-x_{2}\right)\right]+\int_{b_{1}}^{b_{2}} t\left[\frac{1}{2}-x_{1}(b)\left(1-x_{1}(b)\right)\right] d F(b)+F\left(b_{1}\right) \frac{t}{4},
$$

and are minimized when $x_{0}=x_{1}=x_{2}=.5 .^{7}$ Accordingly, any increase in $p_{A}($ above $P / 2)$ increases the level of transportation costs as

$$
\frac{d T C}{d p_{A}}=\frac{s}{2 t}\left[2\left(1-F\left(b_{2}\right)\right)\left(b_{2}-b_{1}\right)+\int_{b_{1}}^{b_{2}}\left(b-b_{1}\right) d F(b)\right]>0 .
$$

Welfare changes with an increase in the asymmetry of law enforcement, using $x_{1}\left(b_{2}\right)=x_{2}$ and $x_{1}\left(b_{1}\right)=.5$, according to

$$
\begin{aligned}
\frac{d W}{d p_{A}}= & f\left(b_{2}\right) s x_{2}\left(h_{A}-p_{A} s\right)-f\left(b_{1}\right) \frac{s}{2}\left(h_{B}-\left(P-p_{A}\right) s\right) \\
& +\frac{s}{2 t}\left[\int_{b_{1}}^{b_{2}}\left(b-h_{B}-v\right) d F(b)+2\left(1-F\left(b_{2}\right)\right)\left(h_{A}-h_{B}\right)\right] \\
& -\frac{s}{2 t}\left[2\left(1-F\left(b_{2}\right)\right)\left(b_{2}-b_{1}\right)+\int_{b_{1}}^{b_{2}}\left(b-b_{1}\right) d F(b)\right]
\end{aligned}
$$

The first term in the first line of (12) illustrates that an increase in $p_{A}$ reduces the number of hardcore criminals at location $\mathrm{A}$, which is socially desirable when there is underdeterrence (i.e., when $h_{A}>p_{A} s$ ). The share of hardcore criminals at location $\mathrm{A}$ is $x_{2} \leq 1 / 2$. The

\footnotetext{
${ }^{7}$ To arrive at (10), note that the transportation costs for some given level of gross benefits for which the indifferent individual is at $y$ are given by $t\left[\int_{0}^{y} x d x+\int_{0}^{1-y} x d x\right]$.
} 
second term in the first line of (12) shows that the consequent reduction in $p_{B}$ draws more individuals into the illegal activity at location $\mathrm{B}$, because the relevant threshold $b_{1}$ is reduced for all individuals with $x \in(1 / 2,1]$ constituting one half of the population. The first term in the second line of (12) shows the welfare effect of turning law-abiding individuals at location A into criminals at location $\mathrm{B}$. The rate $s /(2 t)$ represents the change in the critical level $x_{1}(b)$ (i.e., the marginal effect on the shares of individuals choosing location $\mathrm{A}$ and $\mathrm{B}$ ). The second term in the second line captures the welfare effect of making somebody who offended at location $\mathrm{A}$ offend at location $\mathrm{B}$ (abstracting from the transportation costs). This second term is weighted by $s / t$, that is, the change in the critical level $x_{2}$. The last line of (12) gives the increase in transportation costs that results from an increase in the asymmetry of law enforcement. The third line is necessarily negative. The second line is negative when $h_{A} \approx h_{B}$ and $h_{B}>p_{B} s$. As a result, the addition of the possibility of relocating introduces only additional marginal costs of increasing $p_{A}$ at the expense of $p_{B}$ when $h_{A} \approx h_{B}$.

The level of transportation costs per unit (i.e., $t$ ) scales the importance of the different influences on welfare, and represents the ease with which individuals can switch between locations. Naturally, individuals who undertake the legal activity will always choose the closest location in our setting (given that we abstract from adverse spillovers that may result from high crime rates at a location; see, e.g., Marceau and Mongrain 2011). In contrast, hardcore criminals choose between locations on the basis of both transportation costs and the difference in the expected sanction. We are particularly interested in the extent to which individuals who chose the illegal activity at location A for a uniform (or less asymmetric) law enforcement policy will relocate to location B in order to offend there when the detection probability $p_{A}$ is increased at the cost of the level of $p_{B}$. This possibility is key to assessing the social desirability of focused law enforcement and has been abstracted from in the previous literature.

Figure 2 illustrates the changes in the critical levels that result from a discrete increase in the level of $p_{A}$. The level of $x_{2}$ falls, as does $x_{1}$ for given $b$. Arrow 1 shows the benefit in terms of a higher level of deterrence $b_{2}$. However, this benefit only applies to the small set of individuals for whom $x \leq x_{2}$ holds. Arrow 2 highlights that some people are not deterred 


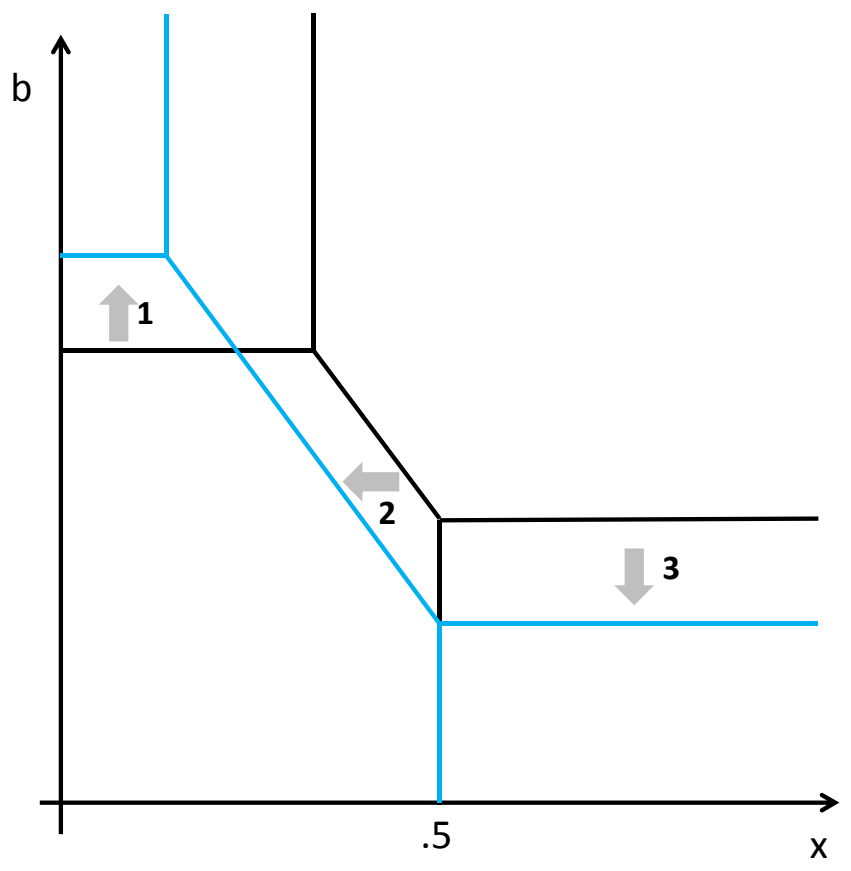

Figure 2: Change in the split of population after discrete increase in $p_{A}$

by the increase in the level of the detection probability in location A, but simply driven to offend at location $\mathrm{B}$ instead. Having $x_{2} \leq x_{1} \leq 1 / 2$ will be all the more important the lower is the level of the transportation cost parameter $t$. The level of $x_{2}$ and the slope of $x_{1}$ in the figure will be small for low $t$. Arrow 3 indicates that the level of deterrence $b_{1}$ falls when $p_{A}$ is increased at the cost of the level of $p_{B}$.

We will now analyze the effects of marginal changes in $p_{A}$. Consider first the value of the derivative of welfare with respect to $p_{A}$ starting from uniform enforcement (i.e., the value of the change in welfare when it is evaluated at $p_{A}=P / 2$ ), such that $b_{1}=b_{2}$ and $x_{0}=x_{1}=x_{2}=1 / 2$. We obtain

$$
\frac{d W(P / 2)}{d p_{A}}=f\left(b_{2}\right) \frac{s}{2}\left(h_{A}-h_{B}\right)+\frac{s}{t}\left(1-F\left(b_{2}\right)\right)\left(h_{A}-h_{B}\right)
$$

The marginal impact on transportation costs at $p_{A}=p_{B}=P / 2$ is zero and thus has no effect 
on the desirability of introducing some asymmetry in detection probabilities. The marginal benefits of increasing the detection probability in location A at the cost of the one in location $\mathrm{B}$ are positive for $p_{A}=P / 2$ if $h_{A}>h_{B}$. The first term in (13) captures the net social gain in terms of reduced harm from increasing the level of deterrence at location $\mathrm{A}$ at the cost of deterrence at location B. The second term captures the diversion of criminals located at $x_{2}=1 / 2$ from location $\mathrm{A}$ to location $\mathrm{B}$ as a result of the introduction of a difference in the expected sanctions. The responsiveness of individuals in this regard is scaled by the level of transportation costs. All remaining terms in (12) do not show when $p_{A}=P / 2$. The expression in (13) allows us to state a result that holds in the limit.

Proposition 1 Some focusing of law enforcement is socially desirable when the act in location $A$ is more harmful than in location $B$.

Our finding is in line with the results of the literature about marginal deterrence (e.g., Shavell 1992). In our analysis, a given act such as speeding may be more harmful in a residential area than in an industrial one, justifying better enforcement in the residential area.

Having $h_{A}>h_{B}$ is a context in which focusing of law enforcement follows quite intuitively. However, the introduction of some focusing of law enforcement may be socially beneficial even when it has no marginal welfare impact in the limit (i.e., when the value of (13) is equal to zero due to $h_{A}=h_{B}=h$ ). To see this, consider only the effect resulting from the influence on the two levels of deterrence (i.e., the first line of (12)) without, at first, taking account of the possibility of relocation. In that scenario, we scrutinize

$$
\frac{s}{2}\left[f\left(b_{2}\right)\left(h-p_{A} s\right)-f\left(b_{1}\right)\left(h-\left(P-p_{A}\right) s\right)\right] .
$$

Expression (14) shows that focusing of law enforcement may result when $h_{A}=h_{B}=h$ only if the density function has a special form. Suppose that $p_{B}=p_{A}-\epsilon$. Then if $f\left(b_{2}\right)=f\left(v+p_{A} s\right)$ is much greater than $f\left(b_{1}\right)=f\left(v+\left(p_{A}-\epsilon\right) s\right)$, this may present a reason to increase $p_{A}$ above $P / 2$ as the additional deterrence in location $\mathrm{A}$ is weighted more heavily than the loss in deterrence in location B. Specifically, the requirement that follows from concentrating on 
the deterrence terms in (12) reads

$$
\frac{f\left(v+p_{A} s\right)}{f\left(v+\left(p_{A}-\epsilon\right) s\right)}>\frac{h-\left(p_{A}-\epsilon\right) s}{h-p_{A} s} .
$$

In (15), the right-hand side is greater than one, already imposing some requirement on the fraction on the left-hand side. Moreover, the argument has so far abstracted from the possibility of relocating, the consideration of which will introduce additional costs into the picture and question the desirability of focusing further. One density function that is regularly used in the literature on the economics of law enforcement for $f(b)$ is the uniform distribution (e.g., Garoupa 1999). We note that it follows from (15) that there will be no focusing of law enforcement for the uniform distribution. ${ }^{8}$

Proposition 2 Uniform law enforcement is socially optimal when both the harm implied by the act is independent of where the offense is committed and the benefits from crime are uniformly distributed.

The analysis up to now identifies both (i) a potential asymmetry in the level of harm imposed on society by a commission of the act in location A and location B and (ii) differences in the magnitude of the density function at various levels of the support $[0, G]$ as justifications for the optimality of some focusing of law enforcement on location A. Regarding the latter, it is intuitive that it may be socially desirable to increase the detection probability in location $\mathrm{A}$ at the cost of the detection probability in location B when there are many (few) individuals responding to the change in deterrence in location A (B). Moreover, the level of the transportation cost parameter $t$ moderates the extent to which these reasons for an asymmetry translate into an actual difference between $p_{A}$ and $p_{B}$.

Proposition 3 Any given focus of law enforcement on location A increases (decreases) with the level of harm $h_{A}\left(h_{B}\right)$ in location $A(B)$ and tends to increase with the level of transportation costs $t$.

\footnotetext{
${ }^{8}$ This can also be seen in (14), because the decisive part of term (15) will be $\left[P-2 p_{A}\right]$.
} 
Proof. When we suppose an interior solution for $p_{A}$ and consider the comparative-statics results for $j, j=h_{A}, h_{B}, t$, it follows that the sign of $d p_{A} / d j$ is the same as that of $d^{2} W / d p_{A} d j$ due to $d^{2} W / d p_{A}^{2}<0$ being required by the sufficient second-order conditions. We find that

$$
\begin{aligned}
\frac{d^{2} W}{d p_{A} d h_{A}}= & x_{2} s f\left(b_{2}\right)+\left(1-F\left(b_{2}\right)\right) \frac{s}{t}>0 \\
\frac{d^{2} W}{d p_{A} d h_{B}}= & -\frac{1}{2} s f\left(b_{1}\right)-\left(1-F\left(b_{2}\right)\right) \frac{s}{t}-\int_{b_{1}}^{b_{2}} \frac{s}{2 t} d F(b)<0 \\
\frac{d^{2} W}{d p_{A} d t}= & \frac{s}{2 t^{2}}\left[\left(b_{2}-b_{1}\right)\left(h_{A}-p_{A} s\right) f\left(b_{2}\right)+\int_{b_{1}}^{b_{2}}\left(h_{B}-\left(P-p_{A}\right) s\right) d F(b)\right] \\
& -\frac{s}{t^{2}}\left(1-F\left(b_{2}\right)\right)\left(h_{A}-h_{B}-\left(b_{2}-b_{1}\right)\right)
\end{aligned}
$$

The derivative in (18) is not unambiguously signed, but we expect that the two underdeterrence terms dominate.

In our analysis, we distinguish two different locations. With regard to the potential use of focused law enforcement, the most striking outcome would be one in which all resources are employed in location A, implying a detection probability of zero at location B. In this case, $p_{A}=P, b_{1}=v<b_{2}=P s+v$, and $x_{0} \leq x_{1}=\frac{1}{2}-\frac{b-v}{2 t}<x_{2}=\frac{1}{2}\left(1-\frac{P s}{t}\right)$, where $(1-P s / t) / 2$ is the number of individuals affected by a marginal increase in $p_{A}$ and is smaller the lower is the level of transportation costs $t$. For $x_{2} \geq 0$, the level of the transportation cost parameter cannot be too small. ${ }^{9}$ We obtain

$$
\begin{aligned}
\frac{d W}{d p_{A}}= & \frac{s}{2}\left[f(P s+v)\left(1-\frac{P s}{t}\right)\left(h_{A}-P s\right)-f(v) h_{B}\right] \\
& +\frac{s}{2 t}\left[2(1-F(P s+v))\left(h_{A}-h_{B}-P s\right)-\int_{v}^{P s+v} h_{B} d F(b)\right]
\end{aligned}
$$

and now seek to understand under what conditions law enforcement resources will be completely focused on location A.

First of all, completely focused law enforcement requires that even the use of all available resources in location A implies a sizable underdeterrence in location A, because $h_{A}-P s>0$ is necessary for the value of (19) to be non-negative. Having a very low level of enforcement resources $P$ available contributes to such a sizable underdeterrence at location A.

\footnotetext{
${ }^{9}$ In fact, $t \geq P s$ must hold, which combined with our initial assumption connotes that $t \in[P s, v / 2)$ describes the possible levels of $t$ for the present considerations.
} 
Second, it may be noted that $(1-P s / t) \rightarrow 0$ for levels of the transportation cost parameter approaching its lower limit. In that case, the value of (19) is likely negative. A small level of the transportation cost parameter makes it less likely that the first term in (19) is positive. In addition, the second term is more important in (19) the smaller is the level of transportation costs, and the second term is negative unless

$$
h_{A}-P s>\frac{\int_{v}^{P s+v} h_{B} d F(b)}{2(1-F(P s+v))}+h_{B} .
$$

This states that the extent of underdeterrence that remains in location A even if all enforcement resources are exclusively used there must surpass the absolute level of harm in location B by a considerable margin. For symmetric levels of harm (i.e., $h_{A}=h_{B}$ ) condition (20) cannot be fulfilled, such that in this case small transportation costs rule out completely focused enforcement.

As argued before, when it is simultaneously true that a crime implies a level of harm that is independent of where it is committed (i.e., when $h_{A}=h_{B}$ ), and the benefits from crime are uniformly distributed, there will be no asymmetry in law enforcement. Condition (21) states the requirement for the first term of (19) turning positive and shows that any asymmetry in harm levels that may justify completely focused law enforcement must indeed be sizable.

$$
\frac{f(P s+v)}{f(v)}>\frac{h_{B}}{\left(h_{A}-P s\right)(1-P s / t)} .
$$

Note the resemblance to (15) used above to argue for the possible move away from uniform enforcement. Imagining that $F$ is a uniform distribution, then condition (21) conveys that the level of underdeterrence in location A must exceed a multiple of the absolute level of harm in location B.

In the following proposition, we summarize our observations for the scenario of completely focused enforcement that are derived from (19):

Proposition 4 (i) Fully focused law enforcement is not socially optimal when the level of $P$ is high enough to make $h_{A}-P s$ moderate.

(ii) Fully focused law enforcement is not socially optimal when the level of transportation 
costs parameter is sufficiently low.

(iii) Fully focused law enforcement is not socially optimal when both the benefits from crime are about evenly distributed and the harm level implied by the act in location $A$ is not too different from that in location $B$.

\subsection{Comparison with Lando and Shavell (2004)}

As noted above, our paper explores possible advantages of focused law enforcement, following the idea laid out in LS. The present setup differs from their model in that potential offenders can choose their location. When individuals incur additional costs in response to law enforcement policy, this should be taken into account when the policy is chosen, which is the basis for our inclusion of transportation costs in the welfare function. For purposes of comparability, however, we will now abstract from transportation costs and set $v=0$ and $h_{A}=h_{B}=h$.

Consider first the case where offenders cannot choose their location (as in LS). Individuals with locations $x \in[0, .5]$ therefore offend (if at all) in location $\mathrm{A}$, and individuals with $x \in(.5,1]$ offend (if at all) at location $\mathrm{B}$, leading to welfare of

$$
w=1 / 2\left[\int_{p_{A} s}^{G}(b-h) d F(b)+\int_{\left(P-p_{A}\right) s}^{G}(b-h) d F(b)\right] .
$$

Following the procedure in LS, we first transform $w$ by subtracting the constant $\int_{0}^{G}(b-$ $h) d F(b)$ and dividing by $p_{i}$ to obtain

$$
\hat{w}=1 / 2\left[p_{A} \frac{\int_{0}^{p_{A} s}(h-b) d F(b)}{p_{A}}+\left(P-p_{A}\right) \frac{\int_{0}^{\left(P-p_{A}\right) s}(h-b) d F(b)}{P-p_{A}}\right] .
$$

where $y_{A}\left(p_{A}\right)=\int_{0}^{p_{A} s}(h-b) d F(b) / p_{A}$ gives the deterrence return per unit of enforcement resource spent at location $\mathrm{A}$.

The central result of LS (their Proposition 2) is as follows: When there is a $p_{A}^{*}$ maximizing $y_{A}$ and requiring all enforcement resources available (i.e., $P \leq p_{A}^{*}$ ), then it should be implemented on $x \in[0, .5]$ even if this comes at the cost of $p_{B}=0$ on $(.5,1]$. For $p_{A}^{*}$, note 
that the term $y_{A}\left(p_{A}\right)$ is maximized at

$$
\left(h-p_{A} s\right) s f\left(b_{2}\right)=\frac{\int_{0}^{p_{A} s}(h-b) d F(b)}{p_{A}},
$$

which is the equivalent of equation (11) in LS. The intuition for their result is that the increase in $p_{A}$ up to $p_{A}^{*}$ maximizes the return per unit of enforcement resources and spreads its use as far as possible ( since $P \leq p_{A}^{*}$ ).

In the remainder of this section, we consider how condition (24) is modified when offenders can choose their locations. Abstracting from $v$ and $T C$ in our specification of welfare, we follow LS and write welfare as

$$
\tilde{w}=1 / 2\left[p_{A} \frac{\int_{0}^{\left(P-p_{A}\right) s}(h-b) d F(b)+\int_{\left(P-p_{A}\right) s}^{p_{A} s} 2 x_{1}(b)(h-b) d F(b)}{p_{A}}+\left(P-p_{A}\right) \frac{\int_{0}^{\left(P-p_{A}\right) s}(h-b) d F(b)}{P-p_{A}}\right],
$$

where

$$
x_{1}(b)=\frac{1}{2}-\frac{b-\left(P-p_{A}\right) s}{2 t} .
$$

The comparison of (23) and (25) clearly indicates the difference in the setups. In our framework, the deterrence benefits from a higher level of $p_{A}$ are scaled down by $x_{1} \leq 1 / 2$ for $b \in \mathcal{C}_{1}$ because offenders with $x \in\left(x_{1}, 1 / 2\right)$ relocate to offend in location B. Concentrating resources in some hot spot is less advantageous from a social standpoint when offenders can easily move somewhere else to undertake their acts. This reality must show in the level of $p_{A}$ that maximizes the deterrence return per enforcement unit. To derive the equivalent of $p_{A}^{*}$, we now consider the level of $p_{A}$ that maximizes the term

$$
\frac{\int_{0}^{\left(P-p_{A}\right) s}(h-b) d F(b)+\int_{\left(P-p_{A}\right) s}^{p_{A} s} 2 x_{1}(b)(h-b) d F(b)}{p_{A}} .
$$

This occurs where

$$
\left(h-p_{A} s\right) 2 x_{2} s f\left(b_{2}\right)-\frac{s}{t} \int_{b_{1}}^{b_{2}}(h-b) d F(b)=\frac{\int_{0}^{b_{1}}(h-b) d F(b)+\int_{b_{1}}^{b_{2}} 2 x_{1}(b)(h-b) d F(b)}{p_{A}} .
$$

The marginal benefits of focusing are reduced in (27) relative to (24) because $x_{2}<1 / 2$ and because the lower $b_{1}$ entices more individuals to become criminals at location $\mathrm{B}$ (as 
represented by the first and second terms, respectively, on the left-hand side). This suggests that the level of $p_{A}$ that maximizes the return per enforcement resource unit in location $\mathrm{A}$ is lower than the one in the absence of the possibility of relocation (although the right-hand side changes also).

The central distinction of our setting relative to the analysis by LS is illustrated in Figure 3 in which the dashed lines represent uniform enforcement. Whereas in the LS setup, the question of focusing law enforcement resources addresses the changes in the critical benefit levels $p_{A} s$ and $p_{B} s$ for a given split of the population (the difference between $b_{1}$ and $b_{2}$ ), our setting includes two additional features of focusing law enforcement resources due to the relocation of offenders: (i) the deterrence benefits from higher $p_{A} s$ apply only to those from the range $[0,1 / 2]$ actually still offending in location A (i.e., individuals with $x \leq x_{2}$ and $b \geq b_{2}$ ), thereby lowering the deterrence impact relative to the LS-setup by the light grey area, and (ii) the reduction in $p_{B} s$ turns lawful citizens from the range $x \in[0,1 / 2]$ into criminals at location B (dark grey area). The importance of the combined grey area is higher the lower is the level of the transportation cost parameter.

\section{Conclusion}

Scarcity of law enforcement resources is a reality in many jurisdictions, which makes their optimal allocation important for welfare. In this context, Lando and Shavell (2004) establish the counterintuitive result that it may be optimal to focus law enforcement resources on one area even if that implies the total neglect of another similar area. This paper complements their contribution and elaborates on their conclusions. Specifically, we analyze the extent to which allowing potential offenders to choose where to conduct crime limits the circumstances under which fully focused law enforcement will result. Our analysis established that focusing law enforcement resources will indeed be much less likely to be socially optimal when criminals can very easily move between locations. 


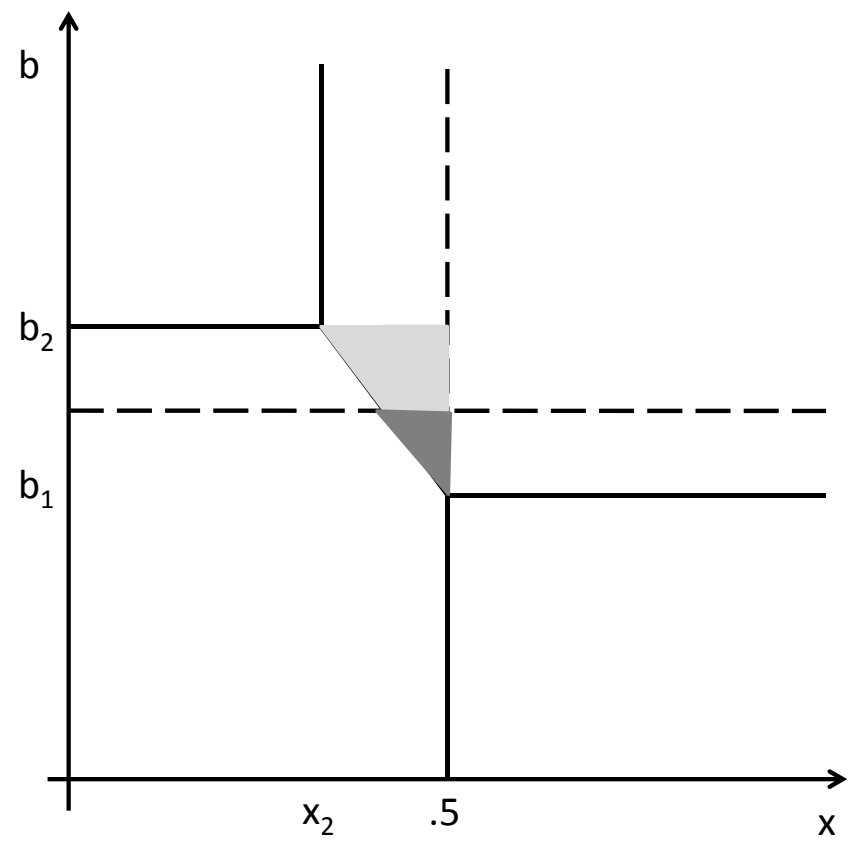

Figure 3: Shaded region highlights additional disadvantages of focusing enforcement

\section{References}

Garoupa, N., 1999. Optimal law enforcement with dissemination of information. European Journal of Law and Economics 7, 183-196.

Hay, B., 2005. Sting operations, undercover cops, and entrapment. Missouri Law Review $70,387-431$.

Kleiman, M.A.R., 2010. When brute force fails: How to have less crime and less punishment. Princeton: Princeton University Press.

Lando, H., and S. Shavell, 2004. The advantage of focusing law enforcement effort. International Review of Law and Economics 24, 209-218.

Marceau, N., 1997. Competition in crime deterrence. Canadian Journal of Economics 30, 844-854. 
Marceau, N., and S. Mongrain, 2011. Competition in law enforcement and capital allocation. Journal of Urban Economics 69, 136-147.

Miceli, T., 2007. Criminal Solicitation, entrapment, and the enforcement of law. International Review of Law and Economics 27, 258-268.

Polinsky, A.M., and S. Shavell, 2007. The theory of public enforcement of law. In: Polinsky, A.M., Shavell, S. (eds.), Handbook of Law and Economics 1. Elsevier, Amsterdam.

Shavell, S., 1992. A note on marginal deterrence. International Review of Law and Economics $12,345-355$. 